\title{
Frenkel-Kontorova 模型非公度结构的动力学
}

献给钱敏教授 90 华诞

\author{
秦文新 \\ 苏州大学数学科学学院, 苏州 215006 \\ E-mail: qinwx@suda.edu.cn \\ 收稿日期: 2017-07-15; 接受日期: 2017-11-07; 网络出版日期: 2017-11-28 \\ 国家自然科学基金 (批准号: 11371270) 资助项目
}

摘要 本文研究 Frenkel-Kontorova (FK) 模型非公度结构的动力学. 所谓非公度结构是指粒子的平均 间距是无理数的情形, 此时系统的相空间是无穷维的. 本文证明在过阻尼条件下, 系统在无穷维相空 间中有一个一维流形, 关于 Poincaré 映射和空间变换群是不变的, 并且此二者在此流形上均诱导出圆 周上的保向同胚. 二者的旋转数分别关联于系统的平均速度和粒子的平均间距.

关键词 Frenkel-Kontorova 模型 非公度结构 单调性

MSC (2010) 主题分类 $34 \mathrm{C} 12,34 \mathrm{C} 15,37 \mathrm{C} 65,37 \mathrm{~L} 60,70 \mathrm{~F} 45$

\section{1 引言}

本文讨论 FK 模型, 也就是粒子链在相互作用势能和周期外势的共同作用下, 在周期力场中的动 力学. FK 模型如下:

$$
\varepsilon \ddot{x}_{n}+\dot{x}_{n}=W^{\prime}\left(x_{n+1}-x_{n}\right)-W^{\prime}\left(x_{n}-x_{n-1}\right)-V^{\prime}\left(x_{n}\right)+F(t), \quad n \in \mathbb{Z},
$$

其中 $x_{n}(t)$ 表示第 $n$ 个粒子的位移, 相互作用势能 $W$ 和外势 $V$ 是 $C^{2}$ 光滑的. 我们始终假定 $W$ 是 严格凸函数, $V$ 是周期函数, 即 $W^{\prime \prime}(x) \geqslant b>0, V(x+1)=V(x), \forall x \in \mathbb{R}$. 另外, $C^{1}$ 光滑的 $T$ - 周期 函数 $F(t)$ 表示周期外力驱动. 对经典的 $\mathrm{FK}$ 模型, $W(x)=k x^{2} / 2, V(x)=\left(1 / 4 \pi^{2}\right) \cos 2 \pi x$, 以及 $F(t)$ $=F+F_{a c} \cos 2 \pi \nu_{0} t$.

在方程组 (1.1) 中, $\varepsilon \ddot{x}_{n}$ 表示惯性项, 本来我们用 $m \ddot{x}_{n}$ 表示, 由于本文中要求质量 $m>0$ 比较小, 因此用 $\varepsilon>0$ 代替.

如果极限

$$
\omega=\lim _{m-n \rightarrow \infty} \frac{x_{m}-x_{n}}{m-n}
$$

存在, 其中 $\boldsymbol{x}=\left(x_{n}\right) \in \mathbb{R}^{\mathbb{Z}}$ 标记粒子的位移, 则称 $\omega$ 是粒子的平均间距. 
设 $\boldsymbol{x}(t)=\left(x_{n}(t)\right)(t \geqslant 0)$ 是系统 (1.1) 的解, 定义极限 (如果存在)

$$
\bar{\nu}=\lim _{\substack{m-k \rightarrow \infty \\ t \rightarrow+\infty}} \frac{1}{t(m-k)} \int_{0}^{t} \sum_{n=k}^{m-1} \dot{x}_{n}(t) d t
$$

为系统的平均速度. 平均速度能够从很多方面反映系统的动力学行为, 是本文的主要研究对象之一.

就我们所知, 与本文相关的关于 (1.1) 的研究结果可综述如下:

若 $W \equiv 0$, 则 (1.1) 描述一个单摆. 这方面的研究成果当然很多. 与本文相关的有文献 $[1,2]$. 这两 篇文献独立地证明了当 $\varepsilon<1 / 4$ (对特定的 $V$ ) 时相应的二阶系统对相空间中定义的某个偏序而言是 单调的, 由此可以得到一系列关于单个振子动力学的结论.

对一般的凸函数 $W$, 可以在构型空间 $\left(\mathbb{R}^{2}\right)^{\mathbb{Z}}$ 中定义一个偏序, 文献 [3] 在过阻尼条件下证明了 (1.1) 是单调的, 并进一步讨论了在常外力驱动情形 (即 $F(t) \equiv F$ ) 和周期边界条件下 (即 $x_{n+q}=x_{n}+p$, 其 中 $p$ 和 $q>0$ 是整数) 系统 (1.1) 的动力学. 对于一般的周期外力驱动, 文献 [4] 讨论了周期边界条件 和过阻尼情形下经典的 FK 模型的 Poincaré 映射的动力学. 文献 [5] 则进一步研究了阻尼系数是非线 性的情形.

我们注意到, 在周期边界条件下, 系统 (1.1) 实际上是一个有限维系统, 文献 [4,5] 充分利用了这 一特点. 另外, 周期边界条件意味着粒子间的平均间距是有理数 $p / q$. 我们的问题是, 当平均间距是无 理数时, 该如何讨论系统 (1.1) 的动力学行为? 此时系统是一个真正的无穷维系统.

文献 [6] 对系统 (1.1) 的非公度结构的动力学 (即平均间距是无理数的情形) 进行了讨论, 不过其 方法只对常外力情形有效, 即 $F(t) \equiv F$. 对周期外力驱动的情形, 文献 [6] 中的方法并不适用. 本文的 目的是讨论系统 (1.1) 在平均间距是无理数情形下 Poincaré 映射的动力学.

\section{2 预备知识}

这部分的内容和记号与文献 [6] 大体相同, 为便于阅读, 简述如下.

$\mathbb{R}^{\mathbb{Z}}$ 中的一个偏序 “ $\leqslant$ ” 定义如下: 设 $\boldsymbol{x}=\left(x_{n}\right), \boldsymbol{y}=\left(y_{n}\right) \in \mathbb{R}^{\mathbb{Z}}$, 如果对任意的 $n \in \mathbb{Z}$, 有 $x_{n} \leqslant y_{n}$, 则称 $\boldsymbol{x} \leqslant \boldsymbol{y}$; 如果 $\boldsymbol{x} \leqslant \boldsymbol{y}$ 且 $\boldsymbol{x} \neq \boldsymbol{y}$, 则称 $\boldsymbol{x}<\boldsymbol{y}$; 如果对任意 $n \in \mathbb{Z}$, 有 $x_{n}<y_{n}$, 则称 $\boldsymbol{x} \ll \boldsymbol{y}$.

令 $\left\{\tau_{j, m} \mid j, m \in \mathbb{Z}\right\}$ 标记 $\mathbb{R}^{\mathbb{Z}}$ 中的变换群

$$
\left(\tau_{j, m} \boldsymbol{x}\right)_{n}=x_{n+j}-m .
$$

如果对任意的 $j, m \in \mathbb{Z}$, 有 $\tau_{j, m} \boldsymbol{x} \leqslant \boldsymbol{x}$ 或者 $\boldsymbol{x} \leqslant \tau_{j, m} \boldsymbol{x}$, 则称 $\boldsymbol{x}$ 是弱旋转有序的; 如果对任意的 $j, m \in \mathbb{Z}$, 有 $\tau_{j, m} \boldsymbol{x} \ll \boldsymbol{x}$ 或者 $\boldsymbol{x} \ll \tau_{j, m} \boldsymbol{x}$ 或者 $\tau_{j, m} \boldsymbol{x}=\boldsymbol{x}$, 则称 $\boldsymbol{x}$ 为旋转有序的. 如果 $\boldsymbol{x}$ 是弱旋转有序的, 则极 限 $\lim _{n \rightarrow \infty} x_{n} / n$ 一定存在, 记为 $\omega$. 此时有性质 $\left|x_{n}-x_{m}-(n-m) \omega\right| \leqslant 1 . \omega$ 称为序列 $\boldsymbol{x}=\left(x_{n}\right)$ 的旋 转数. 如果 $x_{n}$ 标记位移的话, 则 $\omega$ 恰好是平均间距.

将 (1.1) 写成一个一阶系统:

$$
\dot{x}_{n}=v_{n}, \quad \dot{v}_{n}=\left(\frac{1}{\varepsilon}\right)\left[-v_{n}+W^{\prime}\left(x_{n+1}-x_{n}\right)-W^{\prime}\left(x_{n}-x_{n-1}\right)-V^{\prime}\left(x_{n}\right)+F(t)\right], \quad n \in \mathbb{Z} .
$$

令 $\overline{\boldsymbol{x}}=\left(\bar{x}_{n}\right) \in\left(\mathbb{R}^{2}\right)^{\mathbb{Z}}$, 其中 $\bar{x}_{n}=\left(x_{n}, v_{n}\right) \in \mathbb{R}^{2}$, 则 (2.1) 可以简写为

$$
\dot{\overline{\boldsymbol{x}}}=\mathcal{F}(\overline{\boldsymbol{x}}, t) .
$$


在 $\left(\mathbb{R}^{2}\right)^{\mathbb{Z}}$ 中引进范数

$$
\|\overline{\boldsymbol{x}}\|=\sum_{n \in \mathbb{Z}} \frac{\left|x_{n}\right|+\left|v_{n}\right|}{2^{|n|}}
$$

其中 $\overline{\boldsymbol{x}}=\left(\left(x_{n}, v_{n}\right)\right) \in\left(\mathbb{R}^{2}\right)^{\mathbb{Z}}$. 令

$$
X=\left\{\overline{\boldsymbol{x}} \in\left(\mathbb{R}^{2}\right)^{\mathbb{Z}} \mid\|\overline{\boldsymbol{x}}\|<\infty\right\},
$$

则 $X$ 是一个 Banach 空间. 容易验证, 在我们的假设下, $\mathcal{F}: X \times \mathbb{R} \rightarrow X \times \mathbb{R}$ 是局部 Lipschitz 连续的, 因此, (2.2) 的解在 $X$ 中局部存在唯一, 且解对初始条件连续依赖.

定义 $X$ 中的移位变换群 $\left\{\sigma_{j, m} \mid(j, m) \in \mathbb{Z}^{2}\right\}$ :

$$
\left(\sigma_{j, m} \overline{\boldsymbol{x}}\right)_{n}=\left(x_{n+j}-m, v_{n+j}\right), \quad n \in \mathbb{Z} .
$$

如果记 $P^{t} \overline{\boldsymbol{x}}$ 为 $(2.2)$ 以 $\overline{\boldsymbol{x}}$ 为 $t=0$ 时的初始值的解, 则易知 $P^{t}$ 与 $\sigma_{j, m}$ 可交换. 同时, $P^{T}$ 标记 (2.2) 的 Poincaré 映射.

所谓一个系统具有单调性是指, 如果任意两个解在初始时刻可以排序, 则在以后时刻始终保持这 个排序. 由于单调性在我们的讨论中至关重要, 且现在的相空间是二维构型空间 $\left(\mathbb{R}^{2}\right)^{\mathbb{Z}}$ 而不是一维构 型空间 $\mathbb{R}^{\mathbb{Z}}$, 因此, 我们首先要在 $X$ 中定义一个偏序 “ $\leqslant$ ” (参见文献 $\left.[3,6]\right)$. 设 $\overline{\boldsymbol{x}}=\left(\left(x_{n}, \dot{x}_{n}\right)\right), \overline{\boldsymbol{y}}$ $=\left(\left(y_{n}, \dot{y}_{n}\right)\right) \in X$.

定义 1 如果对任意的 $n \in \mathbb{Z}$, 有 $x_{n} \leqslant y_{n}$ 且 $2 \varepsilon \dot{x}_{n}+x_{n} \leqslant 2 \varepsilon \dot{y}_{n}+y_{n}$, 则称 $\overline{\boldsymbol{x}} \leqslant \overline{\boldsymbol{y}}$. 如果 $\overline{\boldsymbol{x}} \leqslant \overline{\boldsymbol{y}}$ 且 $\overline{\boldsymbol{x}} \neq \overline{\boldsymbol{y}}$, 则称 $\overline{\boldsymbol{x}}<\overline{\boldsymbol{y}}$. 如果对任意的 $n \in \mathbb{Z}$, 有 $x_{n}<y_{n}$ 且 $2 \varepsilon \dot{x}_{n}+x_{n}<2 \varepsilon \dot{y}_{n}+y_{n}$, 则称 $\overline{\boldsymbol{x}} \ll \overline{\boldsymbol{y}}$.

定义 2 设 $\overline{\boldsymbol{x}}(t)$ 和 $\overline{\boldsymbol{y}}(t)$ 是系统 (1.1) 的任意两个解. 如果 $\overline{\boldsymbol{x}}(0) \leqslant \overline{\boldsymbol{y}}(0)$ 成立时必有 $\overline{\boldsymbol{x}}(t) \leqslant \overline{\boldsymbol{y}}(t)$, 其中 $t>0$ 在存在区间中, 则称系统 (1.1) 是单调的; 如果 $\overline{\boldsymbol{x}}(0)<\overline{\boldsymbol{y}}(0)$ 成立时必有 $\overline{\boldsymbol{x}}(t) \ll \overline{\boldsymbol{y}}(t)$, 其中 $t>0$ 在存在区间中, 则称系统 (1.1) 是严格单调的.

作一个变量变换 $\Gamma: \overline{\boldsymbol{x}}=\left(\left(x_{n}, v_{n}\right)\right) \in X \mapsto \overline{\boldsymbol{\xi}}=\left(\left(\xi_{n}, \eta_{n}\right)\right) \in X$ :

$$
\xi_{n}=\varepsilon v_{n}+x_{n}, \quad \eta_{n}=\varepsilon v_{n} .
$$

从而, 方程 $(2.2)$ 就写成

$$
\dot{\overline{\boldsymbol{\xi}}}=\Phi(\overline{\boldsymbol{\xi}}, t) .
$$

在新坐标系下, 我们定义偏序 (在不会引起歧义的情况下我们还用记号 $\leqslant$ ) 如下:

$$
\overline{\boldsymbol{\xi}} \leqslant \overline{\boldsymbol{\phi}} \Leftrightarrow \Gamma^{-1}(\overline{\boldsymbol{\xi}}) \leqslant \Gamma^{-1}(\overline{\boldsymbol{\phi}}) .
$$

也就是说, 在新坐标系下, $\overline{\boldsymbol{\xi}}=\left(\left(\xi_{n}, \eta_{n}\right)\right) \leqslant \overline{\boldsymbol{\phi}}=\left(\left(\phi_{n}, \psi_{n}\right)\right)$ 意味着

$$
\xi_{n} \leqslant \phi_{n} \quad \text { 且 } \quad\left|\eta_{n}-\psi_{n}\right| \leqslant\left|\phi_{n}-\xi_{n}\right|, \quad \forall n \in \mathbb{Z} .
$$

对 $\overline{\boldsymbol{\xi}}=\left(\left(\xi_{n}, \eta_{n}\right)\right) \in X, j \in \mathbb{Z}$, 令

$$
m^{+}(j)=\inf \left\{m \in \mathbb{Z} \mid \sigma_{j, m} \overline{\boldsymbol{\xi}} \leqslant \overline{\boldsymbol{\xi}}\right\}, \quad m^{-}(j)=\sup \left\{m \in \mathbb{Z} \mid \overline{\boldsymbol{\xi}} \leqslant \sigma_{j, m} \overline{\boldsymbol{\xi}}\right\} .
$$

对于空集, 理解成 $\inf \emptyset=+\infty$ 和 $\sup \emptyset=-\infty$. 这样就有

$$
m^{-}(j) \leqslant \xi_{n+j}-\xi_{n} \leqslant m^{+}(j), \quad m^{-}(j) \leqslant\left|\eta_{n+j}-\eta_{n}\right|+\xi_{n+j}-\xi_{n} \leqslant m^{+}(j), \quad \forall n, j \in \mathbb{Z} .
$$


定义 3 称 $w(\overline{\boldsymbol{\xi}})=\sup \left\{m^{+}(j)-m^{-}(j) \mid j \in \mathbb{Z}\right\}$ 为 $\overline{\boldsymbol{\xi}}$ 的宽度. 若 $w(\overline{\boldsymbol{\xi}})<\infty$, 则称 $\overline{\boldsymbol{\xi}}$ 有有界宽度.

如果 $(2.2)$ 是单调系统, 利用 $P^{t}$ 与 $\sigma_{j, m}$ 的可交换性可知, 对有界宽度的 $\overline{\boldsymbol{\xi}} \in X$, 有 $w\left(P^{t} \overline{\boldsymbol{\xi}}\right) \leqslant w(\overline{\boldsymbol{\xi}})$, 其中 $t \geqslant 0$ 在定义区间内. 设 $\omega \in \mathbb{R}$. 记

$$
X_{\omega}=\left\{\overline{\boldsymbol{\xi}}=\left(\left(\xi_{n}, \eta_{n}\right)\right) \in X\left|\sup _{n \in \mathbb{Z}}\right| \xi_{n}-n \omega\left|<\infty, \sup _{n \in \mathbb{Z}}\right| \eta_{n} \mid<\infty\right\} .
$$

容易验证, 对任意的 $\overline{\boldsymbol{\xi}}=\left(\left(\xi_{n}, \eta_{n}\right)\right) \in X_{\omega}$ 有有界宽度 $w(\overline{\boldsymbol{\xi}})$, 并且,

$$
\left|\xi_{n+j}-\xi_{n}-j \omega\right| \leqslant w(\overline{\boldsymbol{\xi}}), \quad\left|\eta_{n+j}-\eta_{n}\right| \leqslant w(\overline{\boldsymbol{\xi}}) .
$$

记

$$
X_{\omega}^{1}=\left\{\overline{\boldsymbol{\xi}} \in X_{\omega} \mid w(\overline{\boldsymbol{\xi}}) \leqslant 1\right\} .
$$

本文始终假定 $W$ 和 $V$ 是 $C^{2}$ 光滑的, 满足条件

$$
W^{\prime \prime}(x) \geqslant b>0, \quad V(x+1)=V(x), \quad \forall x \in \mathbb{R} .
$$

又设 $\omega$ 为任意实数. 令

$$
\mu=2 \sup _{|x| \leqslant 1+|\omega|}\left|W^{\prime \prime}(x)\right|+\sup _{x \in[0,1]}\left|V^{\prime \prime}(x)\right| .
$$

命题 1 设 $\mu<1 / 4 \varepsilon$, 则当初始条件 $\overline{\boldsymbol{\xi}}(0) \in X_{\omega}^{1}$ 时系统 $(2.5)$ 的解 $\overline{\boldsymbol{\xi}}(t)$ 在区间 $[0,+\infty)$ 内存在, 并且有 $\overline{\boldsymbol{\xi}}(t) \in X_{\omega}^{1}, \forall t>0$. 进一步, 系统是严格单调的, 即对任意两个解 $\overline{\boldsymbol{\xi}}(t), \overline{\boldsymbol{\phi}}(t) \in X_{\omega}^{1}, t \geqslant 0$, 如果 $\overline{\boldsymbol{\xi}}(0)<\overline{\boldsymbol{\phi}}(0)$, 则 $\overline{\boldsymbol{\xi}}(t) \ll \overline{\boldsymbol{\phi}}(t), \forall t>0$.

证明可参见文献 [6, 引理 2.10] 中的证明. 本文始终假定过阻尼条件 $\mu<1 / 4 \varepsilon$ 成立, 从而系统 $(2.5)$ (即 $(2.2)$ ) 是严格单调的.

注 1 我们看到, 对于经典的 $\mathrm{FK}$ 模型, $W(x)=k x^{2} / 2, k>0$ 和 $V(x)=\left(1 / 4 \pi^{2}\right) \cos 2 \pi x$. 此时 $\mu=2 k+1$ 与 $\omega$ 无关. 过阻尼条件就变成 $\varepsilon<1 / 4(2 k+1)$. 同时, 我们看到, 对 $k=0$, 即单个振子而言, 过阻尼条件就变成 $\varepsilon<1 / 4$. 这就是文献 $[1,2]$ 得到的条件.

定义 4 如果对任意的 $j, m \in \mathbb{Z}$, 有 $\sigma_{j, m} \overline{\boldsymbol{\xi}} \leqslant \overline{\boldsymbol{\xi}}$ 或者 $\overline{\boldsymbol{\xi}} \leqslant \sigma_{j, m} \overline{\boldsymbol{\xi}}$, 则称 $\overline{\boldsymbol{\xi}}$ 是弱旋转有序的; 如果对 任意的 $j, m \in \mathbb{Z}$, 有 $\sigma_{j, m} \overline{\boldsymbol{\xi}} \ll \overline{\boldsymbol{\xi}}$ 或者 $\overline{\boldsymbol{\xi}} \ll \sigma_{j, m} \overline{\boldsymbol{\xi}}$ 或者 $\sigma_{j, m} \overline{\boldsymbol{\xi}}=\overline{\boldsymbol{\xi}}$, 则称 $\overline{\boldsymbol{\xi}}$ 为旋转有序的.

注 $2 \overline{\boldsymbol{\xi}}$ 是弱旋转有序的当且仅当 $w(\overline{\boldsymbol{\xi}}) \leqslant 1$. 另外, 当 $\overline{\boldsymbol{\xi}}=\left(\left(\xi_{n}, \eta_{n}\right)\right) \in X$ 是 (弱) 旋转有序时, 一 维构型 $\boldsymbol{\xi}=\left(\xi_{n}\right) \in \mathbb{R}^{\mathbb{Z}}$ 也是 (弱) 旋转有序的. 因而极限 $\lim _{n \rightarrow \infty} \xi_{n} / n$ 存在.

本文还需要一些 Hausdorff 拓扑的知识, 可以参见文献 [7]. 设 $X$ 是一个距离空间, $A$ 和 $B$ 为 $X$ 中闭集. 定义 Hausdorff 距离为

$$
d(A, B)=\sup \{d(x, B) \mid x \in A\}+\sup \{d(A, y) \mid y \in B\} .
$$

命题 2 紧空间上的闭集的 Hausdorff 距离定义了一个紧拓扑.

\section{3 周期边界条件}

在周期边界条件下, 文献 [4] 对系统 (1.1) 已有一些讨论. 不过需要注意的是, 文献 [4] 中均假定相 互作用势能为 $W(x)=k x^{2} / 2$, 其中 $k>0$ 是常数, 从而是线性耦合, 耦合矩阵是一个实对称矩阵, 除零 
特征值外其余特征值均是负的. 文献 [4] 充分利用了这一特点. 本文中我们要考虑一般的凸相互作用 势能 (即 $\left.W^{\prime \prime}(x) \geqslant b>0\right)$.

系统 (1.1) 的周期边界条件 $x_{n+q}=x_{n}+p$ 等价于 (2.5) 的周期边界条件

$$
\xi_{n+q}=\xi_{n}+p, \quad \eta_{n+q}=\eta_{n}, \quad n \in \mathbb{Z} .
$$

下面对在此周期边界条件下的演化作一个估计. 记 $\overline{\boldsymbol{x}}(t)=\left(\left(x_{n}(t), \dot{x}_{n}(t)\right)\right)(t \geqslant 0)$ 为方程组 $(2.2)$ 的解, 满足周期边界条件 $x_{n+q}(t)=x_{n}(t)+p, t \geqslant 0$, 其中 $p$ 和 $q>0$ 是互素整数. 令

$$
u(t)=\sum_{n=1}^{q} \varepsilon \dot{x}_{n}(t), \quad a=\sup _{x \in[0,1]}\left|V^{\prime}(x)\right|+\sup _{t \in[0, T]}|F(t)| .
$$

引理 1 当 $|u(0)| \leqslant a q \varepsilon$ 时, 对任意 $t>0$, 有 $|u(t)| \leqslant a q \varepsilon$.

证明 在周期边界条件下, 方程组 (1.1) 事实上只有 $q$ 个方程, 将这些方程相加, 得到

$$
-a q \leqslant \dot{u}(t)+\frac{1}{\varepsilon} u(t) \leqslant a q .
$$

由 Gronwall 不等式可得, 当 $|u(0)| \leqslant a q \varepsilon$ 时, 对任意 $t>0$, 有 $|u(t)| \leqslant a q \varepsilon$.

以下我们在新坐标系下讨论 (2.5) 在周期边界条件 (3.1) 下的动力学行为, 其中 $p$ 和 $q>0$ 是整 数. 记 $\overline{\mathbf{1}}=(\ldots,(1,0),(1,0),(1,0), \ldots) \in X$.

定义 5 设 $h: \mathbb{R} \rightarrow X$ 为连续函数, 如果 $h$ 满足 $h\left(s_{1}\right)<h\left(s_{2}\right), \forall s_{1}<s_{2}$, 且 $h(s+1)=h(s)+\overline{\mathbf{1}}$, $\forall s \in \mathbb{R}$, 那么称 $h$ 的像 $\ell=\{h(s) \mid s \in \mathbb{R}\} \subset X$ 为一个有序圆; 如果对任意的 $s_{1}<s_{2}, h$ 还满足 $h\left(s_{1}\right) \ll h\left(s_{2}\right)$, 则称 $\ell$ 为 $X$ 中的一个严格有序圆. $h$ 称为 $\ell$ 的一个参数化.

设 $\ell$ 是一个 (严格) 有序圆, 如果对任意的 $j, m \in \mathbb{Z}$, 有 $\sigma_{j, m} \ell=\ell$, 则称 $\ell$ 对变换群 $\left\{\sigma_{j, m}\right\}$ 是不变 的. 容易验证有序圆 $\ell$ 上的每个元素是弱旋转有序的当且仅当 $\ell$ 关于 $\left\{\sigma_{j, m}\right\}$ 是不变的. 同样地,一个 严格有序圆 $\ell$ 上的每个元素是旋转有序的当且仅当 $\ell$ 关于 $\left\{\sigma_{j, m}\right\}$ 是不变的.

考虑 $X$ 的子空间

$$
X_{p, q}=\left\{\overline{\boldsymbol{\xi}}=\left(\left(\xi_{n}, \eta_{n}\right)\right) \in X \mid \xi_{n+q}=\xi_{n}+p, \eta_{n+q}=\eta_{n}, n \in \mathbb{Z}\right\} .
$$

令 $X_{p, q}^{1}=X_{p / q}^{1} \cap X_{p, q}$. 由命题 1 可知, $P^{T}\left(X_{p, q}^{1}\right) \subset X_{p, q}^{1}$.

定义 6 如果有序圆 $\ell \subset X_{p, q}^{1}$ 的参数化 $h(s)=\left(\left(\xi_{n}(s), \eta_{n}(s)\right)\right)$ 满足条件

$$
\sum_{n=1}^{q} \xi_{n}(s)=q s, \quad\left|\sum_{n=1}^{q} \eta_{n}(s)\right| \leqslant a q \varepsilon
$$

其中常数 $a$ 由 (3.2) 定义, 与 $q$ 无关, 则称 $h$ 为 $\ell$ 的标准参数化.

例如, 取

$$
\xi_{n}(s)=s+\frac{n p}{q}-\frac{p(q+1)}{2 q}, \quad \eta_{n}(s)=0, \quad s \in \mathbb{R},
$$

则 $\ell=\left\{\overline{\boldsymbol{\xi}}=\left(\left(\xi_{n}(s), \eta_{n}(s)\right)\right) \mid s \in \mathbb{R}\right\}$ 是一个关于 $\left\{\sigma_{j, m}\right\}$ 不变的有序圆, 并且 $\ell \subset X_{p, q}^{1}$. 另外, 还注意到 有如下性质: $\sum_{n=1}^{q} \xi_{n}(s)=q s, s \in \mathbb{R}$, 因此, $h(s)=\left(\left(\xi_{n}(s), \eta_{n}(s)\right)\right)$ 是 $\ell$ 的一个标准参数化. 注意到 (3.3) 中的第二个条件显然与引理 1 有关.

引理 2 每个 $X_{p, q}^{1}$ 中的有序圆如果满足 (3.3) 中的第二个条件, 则有唯一的标准参数化. 
引理 2 的证明类似于文献 [8, 引理 3.4] 的证明. 记

$$
\begin{aligned}
& \Omega=\left\{\ell \mid \ell \text { 是 } X_{p, q}^{1} \text { 中关于 }\left\{\sigma_{j, m}\right\} \text { 不变的有序圆, 且满足 }(3.3) \text { 中的第二个条件 }\right\}, \\
& \mathscr{H}=\{h \mid h \text { 是 } \ell \in \Omega \text { 的标准参数化 }\} .
\end{aligned}
$$

对每个 $h:[0,1] \rightarrow X$, 定义

$$
\|h\|_{C^{0}}=\sup _{s \in[0,1]}\|h(s)\| .
$$

容易验证, $\mathscr{H}$ 是一个带有上述 $C^{0}$ 范数的 Banach 空间的非空闭子集. 事实上, 上面的例子表明 $\mathscr{H}$ 是 非空的.

引理 $3 \mathscr{H}$ 是凸集.

证明 设 $\lambda \in[0,1], h, \hat{h} \in \mathscr{H}$. 要验证 $(1-\lambda) h+\lambda \hat{h} \in \mathscr{H}$, 只要验证其对应的有序圆 $\tilde{\ell}$ 关于 $\left\{\sigma_{j, m}\right\}$ 是不变的. 记 $\sigma=\sigma_{1,0}$. 设 $h \in \mathscr{H}$, 则 $\ell=\{h(s) \mid s \in \mathbb{R}\}$ 关于 $\sigma$ 是不变的. 因此, 对任意的 $s \in \mathbb{R}$, 存 在 $s^{\prime} \in \mathbb{R}$, 使得 $\sigma h(s)=h\left(s^{\prime}\right)$, 即 $\xi_{n+1}(s)=\xi_{n}\left(s^{\prime}\right), \forall n \in \mathbb{Z}$. 由周期性和 (3.3) 可知,

$$
q s+p=\xi_{2}(s)+\cdots+\xi_{q+1}(s)=\xi_{1}\left(s^{\prime}\right)+\cdots+\xi_{q}\left(s^{\prime}\right)=q s^{\prime} .
$$

因此有 $s^{\prime}=s+p / q$. 从而, 对任意的 $j, m \in \mathbb{Z}, \sigma_{j, m} h(s)=h(s+j p / q-m), \forall s \in \mathbb{R}$, 对任意 $h \in \mathscr{H}$ 都 成立. 因此有

$$
\sigma_{j, m}[(1-\lambda) h(s)+\lambda \hat{h}(s)]=[(1-\lambda) h+\lambda \hat{h}]\left(s+\frac{j p}{q}-m\right) \in \tilde{\ell},
$$

即 $\sigma_{j, m} \tilde{\ell}=\tilde{\ell}$.

引理 $4 \mathscr{H}$ 是紧集.

证明 设 $h \in \mathscr{H}, h(s)=\left(\left(\xi_{n}(s), \eta_{n}(s)\right)\right)$, 则 $h(s) \in X_{p, q}^{1}$. 由 (2.7) 可知,

$$
\left|\xi_{n+j}(s)-\xi_{n}(s)-\frac{j p}{q}\right| \leqslant 1, \quad\left|\eta_{n+j}(s)-\eta_{n}(s)\right| \leqslant 1, \quad n, j \in \mathbb{Z}, \quad s \in \mathbb{R} .
$$

由此可知,

$$
\left|\xi_{n+1}(s)-\xi_{n}(s)\right| \leqslant 1+\left|\frac{p}{q}\right| \text { 且 }\left|\eta_{n+1}(s)-\eta_{n}(s)\right| \leqslant 1,
$$

以及

$$
\left|\xi_{1}(s)-\xi_{n}(s)\right| \leqslant 1+(n-1)\left|\frac{p}{q}\right|, \quad n=2, \ldots, q, \quad s \in \mathbb{R} .
$$

于是有

$$
\left|q \xi_{1}(s)-\left(\xi_{1}(s)+\cdots+\xi_{q}(s)\right)\right| \leqslant q-1+\frac{q(q-1)}{2}\left|\frac{p}{q}\right|,
$$

从而,

$$
\left|\xi_{1}(s)-s\right| \leqslant \frac{q-1}{q}+\left|\frac{p q(q-1)}{2 q^{2}}\right| \leqslant 1+\frac{|p|}{2},
$$

因此有

$$
\sup _{s \in[0,1]}\left|\xi_{1}(s)\right| \leqslant 2+\frac{|p|}{2} .
$$

同时, 还有

$$
\left|\eta_{1}(s)-\eta_{n}(s)\right| \leqslant 1, \quad n=2, \ldots, q .
$$


可知

$$
\left|q \eta_{1}(s)-\left(\eta_{1}(s)+\cdots+\eta_{q}(s)\right)\right| \leqslant q-1 .
$$

另外, 由于要满足 $(3.3)$ 中的第二个条件, 因此 $\left|\eta_{1}(s)+\cdots+\eta_{q}(s)\right| \leqslant a q \varepsilon$. 从而,

$$
\sup _{s \in[0,1]}\left|\eta_{1}(s)\right| \leqslant \frac{q-1}{q}+\frac{a q \varepsilon}{q} \leqslant 1+a \varepsilon .
$$

同时上述不等式对任意 $\eta_{n}(s)$ 都正确, $n=1, \ldots, q$. 由此可知, 存在常数 $R>0$, 使得对任意的 $h \in \mathscr{H}$, 有 $\|h\|_{C^{0}} \leqslant R$, 因此, $\mathscr{H}$ 是一致有界的. 另外, $\mathscr{H}$ 还是等度连续的.

事实上, 对任意的 $h \in \mathscr{H}, h(s)=\left(\left(\xi_{n}(s), \eta_{n}(s)\right)\right)$, 由 $\xi_{n}$ 的递增性及 (3.3) 中的第一个条件可知, 当 $s_{1}<s_{2}$ 时, 有 $\left|\xi_{n}\left(s_{2}\right)-\xi_{n}\left(s_{1}\right)\right| \leqslant q\left(s_{2}-s_{1}\right)$, 以及由 $h\left(s_{1}\right) \leqslant h\left(s_{2}\right)$ 和 (2.6) 可知,

$$
\left|\eta_{n}\left(s_{2}\right)-\eta_{n}\left(s_{1}\right)\right| \leqslant\left|\xi_{n}\left(s_{2}\right)-\xi_{n}\left(s_{1}\right)\right| \leqslant q\left(s_{2}-s_{1}\right), \quad \forall s_{1}<s_{2}, \quad n=1, \ldots, q .
$$

因此, $h$ 是 Lipschitz 连续的, 且 Lipschitz 常数与 $h$ 无关. 从而 $\mathscr{H}$ 是等度连续的.

由 Arzela-Ascoli 定理可知 $\mathscr{H}$ 是紧集.

利用 Poincaré 映射 $P^{T}$ 可以定义 $\mathscr{H} \rightarrow \mathscr{H}$ 的一个映射: 对每个 $h \in \mathscr{H}$, 令 $g(s)=P^{T} h(s), s \in \mathbb{R}$. 于是由 $P^{T}$ 的严格单调性可知 $g\left(s_{1}\right) \ll g\left(s_{2}\right)$, 对于任意 $s_{1}<s_{2}$ 成立, 另外, 由 (1.1) 向量场的性质可 知, $g(s+1)=g(s)+\overline{\mathbf{1}}$. 再由 $P^{T}$ 与 $\sigma_{j, m}$ 的可交换性可知, $g$ 的像 $\ell^{\prime} \subset X_{p, q}^{1}$ 是关于 $\left\{\sigma_{j, m}\right\}$ 不变的有 序圆. 由引理 1 和 2 知, $\ell^{\prime}$ 有唯一的标准参数化, 记为 $\varphi \in \mathscr{H}$.

定义 $\hat{P}^{T} h=\varphi$. 这样就得到 $\mathscr{H} \rightarrow \mathscr{H}$ 的一个映射 $\hat{P}^{T}$, 并且此映射是连续的. 关于 $\hat{P}^{T}$ 的连续性 证明可以参见文献 [8, 引理 3.6] 的证明. 由于 $\mathscr{H}$ 是非空闭凸紧集, 利用 Schauder 不动点定理可以证 明如下定理:

定理 1 对于任意互素的整数 $p$ 和 $q>0$, 存在一个关于 $\left\{\sigma_{j, m}\right\}$ 和 $P^{T}$ 都不变的严格有序圆 $\ell \subset X_{p, q}^{1}$.

有了前面的准备, 定理 1 的证明可以参见文献 [9, 定理 3.4] 的证明.

\section{4 无理平均间距}

有了周期边界条件 (有理平均间距 $p / q$ ) 的结论, 我们可以利用 Hausdorff 拓扑来讨论无理平均间 距的情形. 设 $-\infty<\alpha<\beta<+\infty$. 记

$$
X_{[\alpha, \beta]}^{1}=\bigcup_{\omega \in[\alpha, \beta]} X_{\omega}^{1}, \quad Y_{[\alpha, \beta]}=\left\{\overline{\boldsymbol{\xi}}=\left(\left(\xi_{n}, \eta_{n}\right)\right) \in X_{[\alpha, \beta]}^{1}|| \eta_{n} \mid \leqslant 1+a \varepsilon\right\} .
$$

引理 $5 \quad Y_{[\alpha, \beta]} /\langle\overline{\mathbf{1}}\rangle$ 是紧集.

引理 5 的证明与文献 [9, 引理 2.12] 的证明类似.

定理 2 对每个无理数 $\omega \in \mathbb{R}$, 存在一个关于 $P^{T}$ 和 $\left\{\sigma_{j, m}\right\}$ 都不变的严格有序圆 $\ell \subset X_{\omega}^{1}$.

证明概要 详细证明参见文献 $\left[9\right.$, 定理 3.5] 的证明. 设 $\omega \in[\alpha, \beta]$ 且有有理数序列 $\omega_{k}=p_{k} / q_{k}$ $\in[\alpha, \beta]$ 收玫到 $\omega$. 令 $\ell_{k}$ 和 $\hat{\ell}_{k}$ 分别标记由定理 1 得到的在 $X_{p_{k}, q_{k}}^{1}$ 和 $X_{p_{k}, q_{k}}^{1} /\langle\overline{\mathbf{1}}\rangle$ 中的严格有序圆. 注意到, 由引理 5 知 $Y_{[\alpha, \beta]} /\langle\overline{\mathbf{1}}\rangle$ 是紧集, 因此其诱导的 Hausdorff 拓扑也是紧的. 另外, 由 (3.5) 知 $\hat{\ell}_{k} \subset Y_{[\alpha, \beta]} /\langle\overline{\mathbf{1}}\rangle$. 因此, $\left\{\hat{\ell}_{k}\right\}$ 是其中的闭集族. 不失一般性, 设在 Hausdorff 拓扑下有 $\hat{\ell}_{k} \rightarrow \hat{\ell}, k \rightarrow \infty$. 因此, $\hat{\ell}$ 是 $Y_{[\alpha, \beta]} /\langle\overline{\mathbf{1}}\rangle$ 中的闭集. 将 $\hat{\ell}$ 周期延拓后的图像记为 $\ell$. 可以证明 $\ell \subset X_{\omega}^{1}$ 是一个关于 $P^{T}$ 和 $\left\{\sigma_{j, m}\right\}$ 都不变的严格有序圆. 
注 3 如果 $(2.5)$ 有一个解 $\overline{\boldsymbol{\xi}}(t)$ 满足 $\overline{\boldsymbol{\xi}}(0) \in \ell \subset X_{\omega}^{1}$, 则 $\overline{\boldsymbol{\xi}}(t) \in X_{\omega}^{1}, \forall t \geqslant 0$. 可以验证在原坐标系 下, 解 $\overline{\boldsymbol{x}}(t)=\Gamma^{-1} \overline{\boldsymbol{\xi}}(t)=\left(\left(x_{n}(t), \dot{x}_{n}(t)\right)\right)$, 有性质

$$
\lim _{n-m \rightarrow \infty} \frac{x_{n}(t)-x_{m}(t)}{n-m}=\omega, \quad \forall t>0,
$$

即 $\overline{\boldsymbol{x}}(t)$ 的平均间距始终是 $\omega, \forall t>0$.

有了定理 2 的结论, 我们就可以考察系统 (1.1) 当粒子的平均间距保持在无理数 $\omega$ 时的动力学. 设 $\ell$ 是由定理 2 得到的严格有序圆. 下面分别考察 Poincaré 映射 $P^{T}$ 和移位变换群 $\left\{\sigma_{j, m}\right\}$ 在 $\ell$ 上的 作用以及由此得到的一些结论.

Poincaré 映射 $P^{T}$ 在 $\ell$ 上的作用就是一个圆周上的保向同胚. 事实上, 设 $h$ 是 $\ell$ 的一个参数 化, 令

$$
G(s)=h^{-1} \circ P^{T} \circ h(s), \quad \forall s \in \mathbb{R} .
$$

可以验证 $G: \mathbb{R} \rightarrow \mathbb{R}$ 满足如下两条性质:

(1) $G$ 严格单调增加, 这可由 $P^{T}$ 的严格单调性以及 $\ell$ 是严格有序圆这一事实得到;

(2) $G(s+1)=G(s)+1$, 这可由 $h(s+1)=h(s)+\overline{\mathbf{1}}$ 以及向量场的性质 ( $V$ 是周期函数) 得到.

$G$ 实际上是一个圆周上保向同胚的提升, 因此存在旋转数

$$
\rho=\rho(G)=\lim _{k \rightarrow \infty} \frac{G^{k}(s)}{k} .
$$

此极限与 $s \in \mathbb{R}$ 无关. 有了此结论, 我们可以证明系统在 $X_{\omega}^{1}$ (甚至在 $X_{\omega}$ 中) 平均速度 $\bar{\nu}$ (见 (1.2)) 是 存在唯一的, 并且有关系式 $\bar{\nu}=\rho / T$, 其中 $T$ 是驱动外力 $F(t)$ 的周期. 证明参见文献 [9].

令 $F=(1 / T) \int_{0}^{T} F(t) d t$, 即 $F$ 是驱动外力的平均值, 则 $F(t)=F+\tilde{F}(t)$, 其中 $\tilde{F}(t)$ 是振动部分. 我们固定振动部分, 变动外力的平均值 $F$, 则平均速度 $\bar{\nu}$ 当然是 $F$ 的函数. 利用系统 $(2.2)$ 的单调性 可以证明 $\bar{\nu}$ 关于 $F$ 是单调增函数, 即对 $F_{1}<F_{2}$, 有 $\bar{\nu}\left(F_{1}\right) \leqslant \bar{\nu}\left(F_{2}\right)$. 另外可以证明 $\bar{\nu}$ 是 $F$ 的连续函数. 这样就存在两个临界值 $F_{d}^{-}(\omega)$ 和 $F_{d}^{+}(\omega)$, 它们当然与平均间距 $\omega$ 有关, 使得当 $F \in\left[F_{d}^{-}(\omega), F_{d}^{+}(\omega)\right]$ 时, $\bar{\nu}=0$; 当 $F<F_{d}^{-}(\omega)$ 时, $\bar{\nu}<0$; 当 $F>F_{d}^{+}(\omega)$ 时, $\bar{\nu}>0$.

如果 $\bar{\nu}=0$, 那么 $\rho=0$. 从而 Poincaré 映射 $P^{T}$ 在 $\ell$ 上有不动点, 由此不动点出发, 就可以得到 原来系统的一个 $T$ 周期解.

下面讨论 $\left\{\sigma_{j, m}\right\}$ 在 $\ell$ 上的作用. 假设 $h(s)=\left(\left(\xi_{n}(s), \eta_{n}(s)\right)\right)$ 是 $\ell$ 的一个参数化, 且满足条件 (这 是可以做到的): $\xi_{0}(s)=s$. 记 $\sigma=\sigma_{1,0}$. 由于 $\sigma \ell=\ell$, 因此对任意的 $s \in \mathbb{R}$, 存在 $s^{\prime} \in \mathbb{R}$, 使得 $\xi_{n}\left(s^{\prime}\right)$ $=\xi_{n+1}(s), n \in \mathbb{Z}$.

取 $n=0,1$, 得到 $s^{\prime}=\xi_{1}(s), \xi_{1}\left(s^{\prime}\right)=\xi_{2}(s)$, 可知 $\xi_{1}\left(s^{\prime}\right)=\xi_{1} \circ \xi_{1}(s)=\xi_{2}(s)$, 因此有 $\xi_{2}(s)=\xi_{1}^{2}(s)$. 事实上可以进一步证明, 对任意正整数 $n$, 有 $\xi_{n}(s)=\xi_{1}^{n}(s), \forall, s \in \mathbb{R}$. 另外, 由于 $\xi_{-1}\left(s^{\prime}\right)=s$, 同时 $s=\xi_{1}^{-1}\left(s^{\prime}\right)$, 从而, $\xi_{-1}(s)=\xi_{1}^{-1}(s), \forall s \in \mathbb{R}$. 因此, 对任意 $n \in \mathbb{Z}$, 有 $\xi_{n}(s)=\xi_{1}^{n}(s), \forall s \in \mathbb{R}$.

又注意到 $\xi_{1}(s+1)=\xi_{1}(s)+1$, 因此 $\xi_{1}$ 实际上是一个圆周保向同胚的提升, 从而存在旋转数. 而 此旋转数恰好就是粒子的平均间距 $\omega$.

事实上, 注意到 $\ell \subset X_{\omega}^{1}$, 因此, 对任意的 $s \in \mathbb{R}$, 由 (2.7) 可知,

$$
\lim _{n \rightarrow \infty} \frac{\xi_{n}(s)-\xi_{0}(s)}{n}=\omega, \text { 因此有 } \lim _{n \rightarrow \infty} \frac{\xi_{1}^{n}(s)-s}{n}=\omega,
$$

即 $\xi_{1}$ 表示的保向圆周同胚的旋转数就是 $\omega$. 至此, 我们知道移位变换 $\sigma$ 在 $\ell$ 诱导出一个圆周上旋转 数为 $\omega$ 的保向同胚, 其旋转数就是粒子的平均间距. 


\section{5 结论与讨论}

由前面的讨论我们得到, 在过阻尼条件下, 当粒子的平均间距是无理数 $\omega$ 时, 以下结论仍然成立 (周期边界情形下的讨论参见文献 $[4,5]$ ):

(1) 系统的平均速度 $\bar{\nu}$ 存在唯一, 即与初始条件 (满足弱旋转有序性) 无关. 另外, $\bar{\nu}$ 关于系统参数 是连续的.

(2) $\bar{\nu}$ 关于驱动外力的平均值 $F$ 是单调增函数, 并且存在临界值 $F_{d}^{-}(\omega) \leqslant F_{d}^{+}(\omega)$, 使得当 $F_{d}^{-}(\omega)$ $\leqslant F \leqslant F_{d}^{+}(\omega)$ 时 $\bar{\nu}=0$.

(3) 当 $\bar{\nu}=0$ 时, 系统 (1.1) 存在平均间距为 $\omega$ 的周期解 $\boldsymbol{x}(t)=\left(x_{n}(t)\right)$ 满足 $x_{n}(t+T)=x_{n}(t)$, $t \in \mathbb{R}, n \in \mathbb{Z}$.

(4) 当 $\bar{\nu} \neq 0$ 且 $\rho=\bar{\nu} T=M / N$ 是有理数时, 其中 $M$ 和 $N>0$ 是互素整数, 则存在平均间距为 $\omega$ 的解 $\boldsymbol{x}(t)=\left(x_{n}(t)\right)$ 满足 $x_{n}(t+N T)=x_{n}(t)+M, t \in \mathbb{R}, n \in \mathbb{Z}$. 如果 $\rho$ 是无理数, 则存在平均间距为 $\omega$ 的拟周期解.

如果我们的模型 (1.1) 描述的是 Josephson 结, 则此时平均速度的物理意义是平均电压. 在我们的 讨论和相关物理文献 $[10,11]$ 中, 系统的平均速度及其性质是关键所在. 在本文及相关文献 $[4,5]$ 中, 我 们是利用相空间中的关于 Poincaré 映射和空间移位变换群不变的有序圆的存在性得到的. 这里有序 圆的概念实际上起源于文献 [1] 中的水平曲线 (horizontal curve) 概念. 其间的差别是有序圆在无穷维 空间 $X$ 中, 而水平曲线在有限维空间中. 另外, 我们也注意到这里的有序圆与文献 $[12,13]$ 中的 ghost circle 十分相像. 事实上, 我们用有理平均间距的有序圆在 Hausdorff 拓扑下取极限这一做法就是得益 于文献 [13] 中的思想.

我们需要提到的是, 当 $\tilde{F}(t) \equiv 0$ 时, 相应的 $F_{d}^{-}(\omega)$ 和 $F_{d}^{+}(\omega)$ 称为脱钉力 (depinning force). 脱钉 力关于系统参数, 特别是关于平均间距 $\omega$ 的连续性问题在文献 [8] 中已有讨论. 并且脱钉力是否为零 还可以作为相应的柱面上的单调扭转映射是否有以 $\omega$ 为旋转数的不变圆的存在性判据 ${ }^{[8]}$. 当 $\tilde{F}(t) \not \equiv 0$ 时, 如上定义的 $F_{d}^{-}(\omega)$ 和 $F_{d}^{+}(\omega)$ 的连续性等问题在本文中没有讨论.

若令 $\varepsilon=0$, 则系统 (1.1) 就退化成一阶系统. 有关此情形数值模拟研究的综述可参见文献 [11]. 有 关此系统在凸相互作用势能下的单调性已有很多研究. 物理学家将此单调性称为禁越规则 (no passing

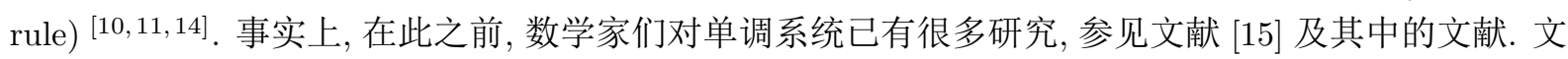
献 [16] 则利用单调系统理论研究了在周期边界条件下常外力驱动情形的动力学. 文献 [17] 讨论了无 理平均间距的情形. 周期外力驱动情形在文献 [4] (周期边界条件) 和 [9] (无理平均间距) 得到讨论.

\section{参考文献}

1 Qian M, Shen W, Zhang J. Global behavior in the dynamical equation of J-J type. J Differential Equations, 1988, 71: 315-333

2 Levi M. Nonchaotic behavior in the Josephson junction. Phys Rev A (3), 1988, 37: 927-931

3 Baesens C, MacKay R S. A novel preserved partial order for cooperative networks of units with overdamped second order dynamics, and application to tilted Frenkel-Kontorova chains. Nonlinearity, 2004, 17: 567-580

$4 \mathrm{Hu}$ B, Qin W X, Zheng Z. Rotation number of the overdamped Frenkel-Kontorova model with ac-driving. Phys D, 2005, 208: $172-190$

5 Qin W X, Peng Z L. Dynamics of the overdamped coupled Josephson junctions with an interference term. J Nonlinear Sci, 2009, 19: 375-398

6 Qin W X. Existence and modulation of uniform sliding states in driven and overdamped particle chains. Comm Math Phys, 2012, 311: 513-538 
7 Katok A, Hasselblatt B. Introduction to the Morden Theory of Dynamical Systems. New York: Cambridge University Press, 1995

8 Qin W X, Wang Y N. Invariant circles and depinning transition. Ergodic Theory Dynam Systems, 2016, doi: http://dx.doi.org/10.1017/etds.2016.42

9 Qin W X. Existence of dynamical hull functions with two variables for the ac-driven Frenkel-Kontorova model. J Differential Equations, 2013, 255: 3472-3490

10 Braun O M, Kivshar Y S. The Frenkel-Kontorova Model, Concepts, Methods, and Applications. Heidelberg: SpringerVerlag, 2004

11 Floría L M, Mazo J J. Dissipative dynamics of the Frenkel-Kontorova model. Adv Phys, 1996, 45: 505-598

12 Golé C. Ghost circles for twist maps. J Differential Equations, 1992, 97: 140-173

13 Golé C. Symplectic Twist Maps: Global Variational Techniques. Singapore: World Scientific Publishing, 2001

14 Middleton A A. Asymptotic uniqueness of the sliding state for charge-density waves. Phys Rev Lett, 1992, 68: 670-673

15 Smith H L. Monotone Dynamical Systems. Providence: Amer Math Soc, 1995

16 Baesens C, MacKay R S. Gradient dynamics of tilted Frenkel-Kontorova models. Nonlinearity, 1998, 11: 949-964

17 Qin W X. Dynamics of the Frenkel-Kontorova model with irrational mean spacing. Nonlinearity, 2010, 23: 1873-1886

\section{Dynamics of incommensurate structures of the Frenkel-Kontorova model}

\section{QIN WenXin}

Abstract In this paper, we study the dynamics of incommensurate structures of the Frenkel-Kontorova model. When the mean spacing of the particles is irrational, we say that the system has incommensurate structures, for which the phase space of the system is infinite-dimensional. We show that under the overdamped condition, there exists a one-dimensional manifold which is invariant both for the Poincaré map and space shifts. Moreover, the Poincaré map and space shifts induce orientation-preserving circle homeomorphisms on this manifold with rotation numbers connecting respectively with the average velocity and the mean spacing.

Keywords Frenkel-Kontorova model, incommensurate structures, monotonicity

MSC(2010) 34C12, 34C15, 37C65, 37L60, 70F45

doi: $10.1360 /$ N012017-00158 